\title{
Glaucoma de pressão normal e espessura corneana central
}

\author{
Normal tension glaucoma and central corneal thickness
}

\author{
Kenji Sakata ${ }^{1}$ \\ Ian Selonke ${ }^{2}$ \\ Artur Schmitt ${ }^{2}$ \\ Alexandre Grandinetti ${ }^{2}$ \\ Viviane Sakata ${ }^{2}$ \\ Daniel R. Guerra ${ }^{3}$ \\ LisandroSakata ${ }^{3}$
}

${ }^{1}$ Chefe do Setor de Glaucoma do Departamento de Oftalmo-Otorrinolaringologia do Hospital das Clínicas da Universidade Federal do Paraná.

${ }^{2}$ Acadêmicos do $6^{\circ}$ ano do curso de Medicina da Universidade Federal do Paraná.

Residente de Oftalmologia do Hospital das Clínicas da Universidade Federal do Paraná.

Endereço para correspondência: R. Itupava, 1428. Alto da Rua XV - Curitiba (PR) CEP 80040-000.

E-mail: knjsakata@hotmail.com

Recebido para publicação em 09.10.01 Aceito para publicação em 04.03.2002

Nota Editorial: Pela análise deste trabalho e por sua anuência na divulgação desta nota, agradecemos ao Dr. Paulo Augusto de Arruda Mello.

\section{RESUMO}

Objetivo: Este estudo tem como objetivo investigar a existência de diferença da espessura corneana central (ECC) em pacientes com glaucoma de pressão normal (GPN), glaucoma crônico simples (GCS) e pacientes normais (Controle). Métodos: Foram avaliados 113 pacientes dos quais 40 apresentavam GPN, 51 eram normais (Controles) e 22 apresentavam GCS. Todos os pacientes foram submetidos a exame oftalmológico completo e tiveram aferidas sua Po(Goldmann)eECC(Topcon SP-2000P). Resultados: A média da espessura corneana central (ECC) dos pacientes com GPN foi de $500,95 \mu$ (d.p. $28,65 \mu$ ), o grupo controle teve média de $521,11 \mu$ (d.p. de $43,30 \mu$ ) e o grupo de GCS apresentou média de $522,23 \mu$ (d.p. de $27,87 \mu$.). O grupo de GPN apresentou uma ECC significativamente menor quando comparado com os grupo controle $(p=0,01)$ e GCS $(p=0,006)$. Entre os grupos controle e GCS não houve diferença estatística significativa ( $p=$ 0,91). Conclusão: Observou-se que numa amostra da população brasileira a ECC dos olhos com GPN é significativamente menor do que a ECC dos pacientes com GCS e do grupo controle.

Descritores: Pressão intra-ocular/fisiologia; Glaucoma/fisiopatologia; Córnea/anatomia \& histologia

\section{INTRODUÇÃO}

A pressão intra-ocular é um importante parâmetro na classificação e acompanhamento dos vários tipos de glaucoma; e a tonometria de aplanação de Goldmann tem sido o método de escolha para a mensuração da pressão intra-ocular $(\mathrm{Po})$ há um longo tempo ${ }^{(1-3)}$. O tonômetro de aplanação de Goldmann mede a Po registrando a força necessária para achatar o ápice corneano, e baseia-se na lei de Imbert-Fick a qual defende que a superfície corneana é perfeitamente elástica e fina, não oferecendo força adicional alguma à aplanação além da pressão interna do globo ocular ${ }^{(4-6)}$. Entretanto ao usar este aparelho estamos sujeitos a alguns erros ${ }^{(3)}$. A grande maioria dos erros pode ser evitada com o uso de uma técnica adequada de mensuração ${ }^{(1)}$, mas a influência da espessura central da córnea na aferição da Po não pode ser evitada. Em seu artigo de lançamento de seu tonômetro de Goldmann, discutiu-se a influência que a espessura central da córnea (ECC) causaria na medida da pressão intra-ocular, mas o autor acreditava que este fator era contrabalançado pela tensão superficial da ponta do tonômetro quando esta toca na córnea ${ }^{(7)}$. Além disso acreditando que variações na ECC não fossem muito comuns ele convencionou como normal uma espessura central da córnea em $0,520 \mathrm{~mm}$ medido através de paquimetria óptica ${ }^{(5)}$. Mas após o surgimento de paquímetros ópticos comercializáveis notou-se que a espessura corneana variava mais do que Goldmann tinha relatado em seus primeiros estudos ${ }^{(5-6)}$. O tonômetro de Goldmann apresenta medidas 
precisas da Po somente quando a espessura corneana é próxima de $0,520 \mathrm{~mm}^{(6,8)}$.

A mensuração da espessura corneana pode ser realizada utilizando dois métodos distintos, o primeiro sendo a paquimetria óptica, e a segunda a paquimetria ultra-sônica (alta reprodutibilidade de resultados); sendo que a primeira técnica apresenta valores menores que a segunda ${ }^{(6)}$.

A ECC é uma variável física que pode alterar a acurácia do tonômetro de Goldmann, e em alguns casos particulares representa um item a mais a ser analisado quando um diagnóstico ou uma pressão alvo está para ser definida.

Este estudo tem como objetivo analisar se existe diferença significativa da ECC entre pacientes com GPN, GCS e normais em uma população brasileira.

\section{MÉTODOS}

Este estudo foi realizado de janeiro de 1999 a dezembro de 2000 e faz parte do protocolo do projeto de Extensão Universitária - Projeto Glaucoma. A população estudada compreendeu um total de 113 pacientes sendo dividida em três grupos: 1Glaucoma de pressão normal $(n=40) ; 2$ - Controle $(n=51)$ e 3 Glaucoma crônico simples $(n=22)$, os quais foram avaliados no ambulatório de Oftalmologia do Hospital de Clínicas da Universidade Federal do Paraná. Os pacientes com glaucoma de pressão normal tiveram seu diagnóstico confirmado no Projeto Glaucoma e então, no momento do exame não estavam utilizando nenhuma medicação antiglaucomatosa. Os pacientes do grupo glaucoma crônico simples eram pacientes do ambulatório de Glaucoma do Hospital de Clinicas e já se encontravam em tratamento com um ou mais das seguintes medicações: $\beta$-agonistas; $\alpha$-agonistas, prostaglandinas.

As distribuições por sexo e idade nos 3 grupos estão descritas na tabela 1 .

Os pacientes incluídos no grupo GPN apresentaram medida da pressão intra-ocular $(\mathrm{Po})$ menor do que $21 \mathrm{mmHg}$, a qual foi aferida pelo menos 2 vezes em ocasiões diferentes pelo tonômetro de aplanação de Goldmann (marca Haag Streit ${ }^{\circledR}$ ) acoplado à lâmpada de fenda; gonioscopia demonstrando seio camerular aberto e normal; alterações de campo visual e fundoscopia compatíveis com lesões glaucomatosas sem apresentar nenhuma evidência de outras patologias que justificassem o quadro clínico.

\begin{tabular}{|c|c|c|c|c|c|c|}
\hline & M. Idade & V. Mín. & V. Máx. & d. p. & Homens & Mulheres \\
\hline GPN & 55,48 & 32 & 82 & 11,58 & $24(60 \%)$ & $16(40 \%)$ \\
\hline Controle & 49,70 & 20 & 77 & 10,79 & $29(57 \%)$ & 22 (43\%) \\
\hline GCS & 55,36 & 39 & 75 & 11,17 & $8(36 \%)$ & $14(64 \%)$ \\
\hline
\end{tabular}

Os pacientes inclusos no grupo GCS apresentaram medida da pressão intra-ocular (Po) maior do que $21 \mathrm{mmHg}$, a qual foi aferida pelo menos 2 vezes em ocasiões diferentes pelo tonômetro de aplanação de Goldmann; alterações de campo visual, escavação do nervo óptico compatível com lesão glaucomatosa e gonioscopia demonstrando seio camerular aberto e normal.

A campimetria foi realizada através do perímetro computadorizado TOPCON modelo SBP 2020, estratégia 24/2.

Os critérios adotados como suspeito de glaucoma pela fundoscopia foram: uma escavação maior que 0,5 ; a escavação vertical maior que a horizontal; a assimetria de escavação maior que 0,2 ; a presença de hemorragias no disco óptico e a presença de "notch". Adotaram-se os seguintes critérios para o diagnóstico de glaucoma na perimetria: escotoma de Seidel; depressão nasal superior ou inferior; degrau nasal; escotomas arqueados superiores ou inferiores completos ou incompletos.

Foram excluídos do estudo pacientes com historia pregressa de cirurgia ocular, miras irregulares à tonometria de aplanação (córneas e filmes lacrimais normais) ou astigmatismo maior do que 4 dioptrias $^{(9-10)}$.

Todos os olhos tiveram a pressão intra-ocular (Po) aferida através do tonômetro de aplanação de Goldmann modelo Haag Streit, e a espessura corneana central (ECC) mensurada com o paquímetro óptico de marca Topcon modelo SP-2000P.

$\mathrm{Na}$ aferição da Po foram realizadas 3 medidas por um mesmo examinador (um de quatro examinadores) obtendo-se a média entre elas. Procedeu-se da mesma forma para a aferição da ECC.

Neste estudo houve mascaramento do diagnóstico, entretanto não houve mascaramento das medidas pelos examinadores; mas os mesmos não estavam cientes da realização deste estudo no momento do atendimento.

Para análises estatísticas, foi selecionado aleatoriamente apenas um olho de cada paciente.

\section{RESULTADOS}

Conforme demonstrado na tabela 2, a Po no grupo GPN teve uma média de $14,73 \mathrm{mmHg}(\mathrm{dp}=3,02 \mathrm{mmHg})$. No grupo dos pacientes controle a média da Po foi de $15,62 \mathrm{mmHg}(\mathrm{dp}=$ $3,35 \mathrm{mmHg}$ ). No grupo GCS a média da Po foi de 19,91 $\mathrm{mmHg}$ $(\mathrm{dp}=3,10 \mathrm{mmHg})$.

Conforme a tabela 3, a Espessura Central Corneana (ECC) no grupo GPN (GPN) teve uma média de 500,95 $\mu \mathrm{m}$

\begin{tabular}{|lcccc|}
\hline \multicolumn{4}{|c|}{ Tabela 2. Valores das médias da Po nos três grupos } \\
Po (mmHg) & Média & $\begin{array}{c}\text { Valor } \\
\text { Mínimo }\end{array}$ & $\begin{array}{c}\text { Valor } \\
\text { Máximo }\end{array}$ & $\begin{array}{c}\text { Desvio } \\
\text { Padrão }\end{array}$ \\
Grupo GPN & 14,73 & 8 & 21 & 3,02 \\
Grupo Controle & 15,62 & 9 & 21 & 3,35 \\
Grupo GCS & 19,91 & 15 & 27 & 3,10 \\
Po-Pressão intra-ocular;GPN-Glaucoma de pressão normal;GCS-Glaucoma \\
crônico simples
\end{tabular}




\begin{tabular}{|lcccc|}
\hline \multicolumn{4}{|c|}{ Tabela 3. Valores das médias da ECC nos três grupos } \\
ECC $(\mu)$ & Média & $\begin{array}{c}\text { Valor } \\
\text { Mínimo }\end{array}$ & $\begin{array}{c}\text { Valor } \\
\text { Máximo }\end{array}$ & $\begin{array}{c}\text { Desvio } \\
\text { Padrão }\end{array}$ \\
Grupo GPN & 500,95 & 428 & 563 & 28,65 \\
Grupo Controle & 521,11 & 436 & 617 & 43,30 \\
Grupo GCS & 522,23 & 471 & 576 & 27,87 \\
ECC - Espessura corneana central; GPN - Glaucoma de pressão normal; \\
GCS - Glaucoma crônico simples
\end{tabular}

$(\mathrm{dp}=28,65 \mu \mathrm{m})$. No grupo controle a média da ECC foi de $521,11 \mu \mathrm{m}(\mathrm{dp}=43,30 \mu \mathrm{m})$. No grupo GCS a média foi de $522,23 \mu \mathrm{m}(\mathrm{dp}=27,87 \mu \mathrm{m})$.

Foi realizada a análise das variâncias com intervalo de confiança de $95 \%$ que demonstrou que a ECC é significativamente menor no grupo GPN comparado ao grupo controle ( $\mathrm{p}=$ $0,01)$ e grupo GCS $(p=0,006)$. Foi também realizada análise das variâncias com intervalo de confiança de $95 \%$ entre o grupo controle e o grupo de GCS a qual não demonstrou diferença estatisticamente significativa $(\mathrm{p}=0,91)$.

Também foi realizado o cálculo da regressão linear da Po x ECC nos três grupos, mas falhou em encontrar uma correlação estatisticamente significativa em todos eles. No grupo GPN: $\mathrm{r}=0,25875$ e um $\mathrm{p}=0,10692 ;$ no grupo Controle: $\mathrm{r}=0,13283 \mathrm{e}$ um $\mathrm{p}=0,37344$; e no grupo GCS: $\mathrm{r}=0,29455$ e um $\mathrm{p}=0,18331$.

\section{DISCUSSÃO}

A tonometria de aplanação de Goldmann é o método mais utilizado para a medida da pressão intra-ocular. Entretanto, dentre as várias fontes de erro na leitura, a espessura da córnea é um fator físico importante que influencia na rigidez corneana e, então na aferição da Po medida pelo tonômetro de aplanação(4).

Revisando a literatura, encontra-se em trabalho publicado em 1999 que não encontrou diferença estatística na espessura da córnea entre pacientes normais $(552 \pm 35 \mu \mathrm{m})$ e com glaucoma crônico simples $(543 \pm 35 \mu \mathrm{m})$, mas o grupo formado pelos pacientes com glaucoma de pressão normal $(521 \pm 31 \mu \mathrm{m}) \mathrm{em}$ relação aos do grupo do GCS e grupo controle apresentavam uma espessura corneana significativamente menor $(p=0,001$; $p=0,001)$. Utilizando-se de um fator de correção da Po pela espessura corneana o autor reclassificou $31 \%$ dos pacientes com GPN para Glaucoma crônico simples ${ }^{(2)}$. Em outro trabalho com resultados semelhantes ao anterior, encontrou-se uma ECC no grupo GPN $(521 \pm 37 \mu \mathrm{m})$; no GCS $(536 \pm 35 \mu \mathrm{m})$ com $\mathrm{p}=$ 0,0028 e para o grupo controle $(556 \pm 34 \mu \mathrm{m})$ com um $\mathrm{p}=$ $0,0037^{(4)}$.

No entanto, em estudo publicado em dezembro de 2000, não foi constatada diferença estatisticamente significativa da ECC entre seus três grupos analisados. Seus achados foram, para GPN $(552 \pm 36 \mu \mathrm{m}) ;$ GCS $(550 \pm 32 \mu \mathrm{m})$ e GPN $(544 \pm$
$33 \mu \mathrm{m})^{(6)}$. Nestes três últimos artigos citados os autores utilizaram o paquímetro ultra-sônico.

Na prática clínica, a ECC se apresenta como um dos importantes dados a serem analisados no diferencial de casos de hipertensão ocular e glaucoma crônico simples, visto que existe na literatura trabalhos que comprovam que a ECC é significativamente maior nos pacientes com hipertensão ocular ${ }^{(1-2,5-7)}$.

O diagnóstico diferencial entre GCS e GPN (ainda que alguns autores relatem diferenças na evolução, na faixa etária acometida, alterações no campo visual e na fundoscopia e até mesmo sugiram fisiopatologias diferentes) ${ }^{(11)}$ é realizado baseado na Po acima ou abaixo do consenso de limite de Po normal, $21 \mathrm{mmHg}$, sendo que a Curva Tensional Diária classificaria corretamente pacientes com GPN ou GCS ${ }^{(12)}$. Então, ainda que controverso na literatura, a ECC poderia ser responsável pela alteração do diagnóstico de GPN para GCS naqueles casos que apresentam Po limítrofes e ECC fina. Esta classificação correta tem sua importância visto que muitos glaucomatólogos atualmente acreditam que o fator mecânico exerça um papel com menor importância nos casos de GPN, sendo que outros fatores (neurológico, vascular, genético) assumam uma maior importância no desenvolvimento da lesão glaucomatosa.

Ainda discutindo sobre a importância clinica, a Po ainda é o único fator comprovado passível de tratamento, seja por condutas clinicas ou cirúrgicas. Então, novamente, a ECC deve ser considerada como um dos itens a serem analisados nos casos em que exista uma progressão do GPN apesar da pressão alvo ter sido aparentemente atingida; principalmente quando nestes casos está se discutindo a indicação de condutas mais agressivas. Já nos pacientes de GPN que irão iniciar seu tratamento, deve-se considerar a ECC antes de definir a pressão alvo.

O fato de não existir uma correlação linear estatisticamente significativa nos três grupos não representa uma evidência contra a influência da ECC na aferição da Po, mas sim uma falha em se quantificar esta influência. Isto porque cada paciente apresenta uma Po específica e esta não é definida única e exclusivamente pela ECC, e sim por um conjunto de fatores. Então, a correlação direta da Po e ECC não pode ser realizada sem se conhecer a real Po de cada paciente. Portanto para se encontrar uma equação de correção da Po pela ECC, necessitar-se-ia, por exemplo, de um estudo com uma quantidade estatisticamente suficiente de pacientes que apresentassem uma Po aferida manometricamente (paracentese acoplada a um manômetro) iguais (ex. $15 \mathrm{mmHg}$ ) e que então, tivessem sua Po (Goldmann) e ECC aferidas para daí então ser realizado o cálculo da correlação linear.

\section{CONCLUSÃO}

Neste estudo realizado numa amostra da população brasileira, a ECC do grupo GPN se apresentou significativamente menor que nos grupos controle e GCS. 


\section{ABSTRACT}

Purpose: To determine if there is a significant difference in central corneal thickness (CCT) among patients with normal tension glaucoma (NTG), primary open angle glaucoma (POAG) and normal patients. Methods: One hundred and thirteen patients were evaluated; 40 had NTG, 51 were normal and 22 had POAG. IOP (Goldmann) and CCT (Topcon SP2000P) were measured in all patients. Results: The CCT mean in NTG patients was $500.95 \mu(\mathrm{SD}=28.65 \mu)$; in normal patients the CCT mean was $521.11 \mu(\mathrm{SD}=43.30 \mu)$; and in the POAG group the CCT mean was $522.23 \mu(\mathrm{SD}=27.87 \mu)$. By statistical analysis (ANOVA, 95\% confidence interval) the CCT in NTG was significantly lower than in normal patients and in the POAG group $(p=0.01$ and $p=0.006)$, but no differences was found between normal patients and the POAG group $(p=0.91)$. Conclusions: In this study we concluded that in a Brazilian population sample the NTG patients tend to present significantly lower ECC measurements when compared with normal and POAG patients.

Keywords: Intraocular pressure/physiology; Glaucoma/ physiopathology; Cornea/anatomy \& histology

\section{REFERÊNCIAS}

1. Wolfs RC, Klaver CC, Vingerling JR, Grobbee DE, Hofman A, de Jong PT. Distribution of central corneal thickness and its association with intra-ocular pressure: The Rotterdam Study. Am J Ophthalmol 1997;123:767-72.

2. Copt RP, Thomas R, Mermoud A. Corneal thickness in ocular hypertension, primary open-angle glaucoma, and normal tension glaucoma. Arch Ophthalmol 1999;117:14-6.

3. Sakata K, Figueira ALM, Guimarães ACP, Schmitt AJ, Scapucin L, Barros LGR, Delai N. Estudo da correlação entre pressão intra-ocular e espessura corneana central (Projeto glaucoma). Arq Bras Oftalmol 2000;63:355-8.

4. Morad Y, Sharon E, Hefetz L, Nemet P. Corneal thickness and curvature in normal tension glaucoma. Am J Ophthalmol 1998;125:164-8.

5. Herndon LW, Choudhri SA, Cox T, Damji KF, Shields MB, Allingham RR. Central corneal thickness in normal, glaucomatous and ocular hypertensive eyes. Arch Ophthalmol 1997;115:1137-41.

6. Wu LL, Suzuki Y, Ideta R, Araie M. Central corneal thickness of normal tension glaucoma patients in Japan. Jpn J Ophthalmol 2000;44:643-7.

7. Argus WA. Ocular hypertension and central corneal thickness. Ophthalmology 1995;102:1810-2.

8. Whitacre MM, Stein RA, Kassanein K. The effect of corneal thickness on applanation tonometry. Am J Ophthalmol 1993;115:592-6.

9. Shields MB. Glaucoma: A pressão intra-ocular. São Paulo: Panamericana; 1989. p.47-71

10. Mark HH. Corneal curvature in applanation tonometry. Am J Ophthalmol 1973;76:223-4.

11. Werner EB. Normal tension glaucoma. In: Ritch R, Shields MB, Krupin T. The glaucomas. $2^{0}$ ed. St. Louis: Mosby; 1989. p.769-97.

12. Calixto N, Meira DM, Cronemberger S. Estudo de pacientes com suspeita diagnóstica de glaucoma de pressão normal. Rev Bras Oftalmol 1997;56:823-34.

\title{
1 Simpósio Infernacional de Retinoblastoma
}

\author{
(i e $z$ de Mlarç̧o de 2003 \\ Hotel Royyal Palın Playza - Cammpinas - SPP
}

Pronnoção: Centrơ Infiantil Boldrini

INFORMAÇÕES: Tel.: (19) 3787-5031/3787-5115

E-mail: simposio2003@boldrini.org.br 